\title{
Téoros
}

Revue de recherche en tourisme

\section{Des outils et des intervenants}

\section{Yves Archambault}

Volume 2, numéro 3, octobre 1983

Les multiples facettes de l'aménagement touristique

URI : https://id.erudit.org/iderudit/1080822ar

DOI : https://doi.org/10.7202/1080822ar

Aller au sommaire du numéro

Éditeur(s)

Université du Québec à Montréal

ISSN

0712-8657 (imprimé)

1923-2705 (numérique)

Découvrir la revue

Citer cet article

Archambault, Y. (1983). Des outils et des intervenants. Téoros, 2(3), 2-5.

https://doi.org/10.7202/1080822ar d'utilisation que vous pouvez consulter en ligne.

https://apropos.erudit.org/fr/usagers/politique-dutilisation/ 


\section{Des outils et des intervenants}

Par Yves Archambault

Premiere étape: notre ami se présente au Service de l'hotellerie du ministere de IIndustrie, du Commerce et du Tourisme, où un technicien l'accueille aimablement. Ce spécialiste examine avec soin les plans du projet. On lui indique les modifications a apporter afin de satisfaire aux exigences des reglements; pour ce travail il doit debourser une legere somme par mandat ou cheque vise.

Notre ami, ne sachant pas qu'il devait obtenir une approbation du ministere du Travail, repart allegrement vers ces lieux tout aussi aseptisés. Ah ouil il a oublié aussi les titres de propriété du terrain où il compte réaliser son projet. En passant, aussi bien se procurer le certificat d'acceptation émis par sa ville ou sa municipalité, ça fait partie des exigences réglementaires. Important: le technicien hi a bien mentionné de se rendre au ministère de l'Environnement puisque les services d'aqueduc et d'egout ne sont pas fournis par sa ville; il doit avoir en main le certificat de ce ministere.

Petit délai iciet la, modifications mineures au plan, retouches par ci, parlà, bref cinq à six semaines apres, notre ami, fier de ses performances, rebondit au Service de /hótellerie où il obtient enfin son certificat d'amenagement.

Entretemps, il croit avantageux de se prévaloir des avantages de la loi de l'aide au développement (le Credit touristique). Muni de son dossier, garni de certificats de toutes les couleurs. il doit d'abord faire une demande par écrit pour recevoir les docu. ments nécessaires a la demande de credit.

Ul reçoit, tel que prévu, des formules à completer. Rien de compliqué, le tout est retourné au service concerné qui delegue un analyste touristique. Autour d'un bon repas, analyste et promoteur discutent affaires. Pendant que le dossier chemine, la construction démarre, les inspecteurs se suivent et se ressemblent, /avenir est prometteur.
Offrant le gite et le repas, notre ami doit aussi se procurer les permis nécessaires. Rien de plus simple, il $y$ en a seulement trois:

- un pour exploiter san hótel et sa salle à manger.

- un pour exploiter son camping.

- un pour son service de boisson.

De toute façon, pour obtenir le crédit touristique, il doit arborer toutes ces médailles. Au fait, un nouvel analyste se presente, celui de la Societé de Développement Industriel. Celui-la fait une etude du portefeuille de notre homme: sa solvabilité semble bonne, il pourra obtenir un prêt et une prise en charge, terme d'un fonctionnarisme ronronnant qui signifie tout bonnement une subvention.

Après quelques mois, la construction est terminée, c'est le moment d'obtenir les trois permis. I/ fallait bien attendre, car les inspecteurs doivent voir les travaux lorsque terminés. Heureusement pour notre cher ami, tout est conforme: la sécurité, les superficies, les volumes d'air, l'électricité, les égoúts, lapprovisionnement en eau, la cuisine, le garde-manger, la chambre froide, les lumieres de sortie, les portes paniques, les stationnements, les accés, etc.

Cet extrait de l'excellent texte de Jacques Demers ${ }^{\text {(1) }}$ illustre de façon très imagée. mais combien convaincante la kirielle de fonctionnaires, d'agences gouvernementa les, de règlements et autres obstacles aux. quels tout promoteur public ou privé devra se heurter avant de pouvoir réaliser un projet d'amenagement touristique.

De tous les intervenants en matière d'amé nagements touristiques, les plus connus du public sont certes les organismes dont la fonction est essentiellement reliée au domaine, tels le ministère du Loisir, de la Chasse et de la Pêche ou Parcs Canada.
On est fin prêt pour cette derniere et sublime etape des trois permis. Le cycle recommence: titres, certificats, preuves municipales, environnementales, travaux, en plus des prix des chambres et des unités de camping. Ahl le permis de la Société des alcools: le pas de danse est différent. il y a un peu plus de contorsions, c'est le tango: requéte, enquête, tribunal, ardonnance... le permis.

Enfin c'est fini... ou presque, la réception d'ouverture a lieu tout juste avant la sajson touristique; fonctionnaires, analystes, député, maire, échevins saluent la nouvelle entreprise et sabrent le champagne en attendant le permis de la Société des alcools... Malediction, dans moins de quelques semaines, il faut renouveler les permis! C'est la valse à quatre temps.

Voila, d'une façon imagee mais combien véridique, le passage à la dowane gouver. nementale pour le promoteur touristique. Attention, i'ai omis deux lois importantes, la loi 125 sur /aménagement et f'urbanisme et la loi 90 sur le territoire agricole. 
Traditionnellement, par le passé, ces actions de planification étaient l'objet de décisions à la pièce et, si elles répondaient à un réel besoin, elles ne répondaient pas nécessairement à des impératifs rigoureux, tant sur le plan des priorités que sur celui de l'opportunité. La situation évolue, toutefois, puisque de nouveaux outils et mécanismes sont maintenant en place qui laissent entrevoir une meilleure concertation en matière d'aménagement.

Avant de traiter de ces nouveaux outils que constituent notamment la Loi sur la protection du territoire agricole et la Loi sur l'amé nagement et l'urbanisme, il n'est pas inutile de passer en revue les differents types d'aménagements touristiques selon une typologie développée en fonction de la pla nification. Dans une deuxième partie, nous aborderons le rỏle des intervenants en rela tion avec ces differents types d'aménagements.

\section{Une typologie des interventions ou aménagements}

Si l'on tente de catégoriser les différents types d'aménagernents susceptibles d'intéresser les touristes, l'on peut au départ, sur le plan de la planification, les classer sous deux grandes catégories: ceux qui sont concus pour les touristes ou en fonction du tourisme, et les autres. La première catégorie regroupe non seulement les projets à caractère proprement touristique, mais également tous ceux dont la conception a tenu compte de la dimension touristique. Quant a la deuxième categorie, elle comprend tous les autres types d'aménagements qui, de par la nature même du touriste, ce bipède curieux, se retrouvent bien malgré eux dans la catégorie des attractions touristiques. II va de soi que la préoccupation de la clientèle touristique variera énormément $d^{\prime} u n$ type de projet à un autre. Voyons de plus près les implications de ce découpage sur le plan de la planification.

Les "vrais aménagements touristiques" Cette catégorie englobe tous ces projets qui n'ont d'autre raison d'être que de procurer une "destination touristique". Se retrouvent dans cette catégorie tous les projets qui sont liés à une attraction naturelle et dont on tire parti. Ces attractions sont intimement rattachées à un espace géographique déterminé, et leur succès dépend d'un équilibre entre divers facteurs, tels l'accessibilité et le coût, mis en relation avec le degré de satisfaction engendrée. Même si elles comptent parmi les plus belles plages qui se puissent rencontrer, celles des lles de la Madeleine, par exemple, demeureront toujours sous-exploitées, en raison de leur éloignement certes, mais également à cause de cette equation plage $=$ chaleur que l'on retrouve plutót rarement aux lles.

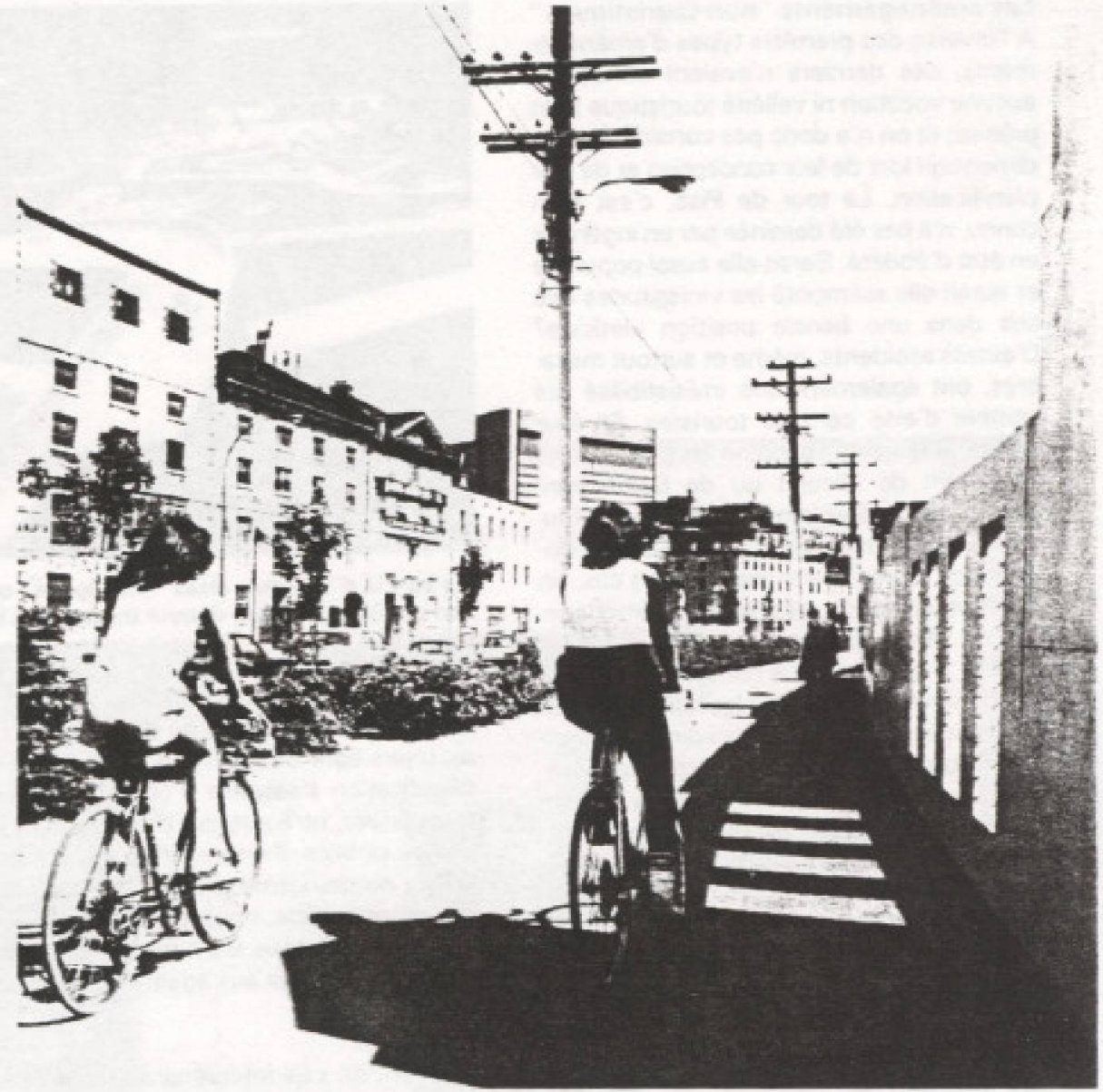

Le Vieux Port de Montréal: une fenêtre sur le fleuve!

Une sous-catégorie englobe les entreprises qui visent soi-disant a "bonifier" un lieu touristique reposant sur des attraits naturels. Pourquoi un musée de l'auto à Niagara Falls? Sürement pas pour attirer les touristes, les Chutes sont là pour celà! mais bel et bien justement parce que les Chutes attirent les touristes en abondance. II s'agit la d'activités dites "foot loose" que l'on pourrait donc à la rigueur implanter n'importe où, mais qui se retrouvent pour la plupart greffées a d'autres installations dont elles deviennent en quelque sorte des parasites. Ces activités de type "foot loose" ne sont pas toutes parasitaires ni reliées necessairement à des attractions naturelles. Le Parc Safari Atricain d'Hemmingford ou le Zoo de Granby servent au contraire de points d'ancrage à d'autres activités qui benéficient ainsi du pouvoir d'attraction des précéden tes lun musee de l'auto a Granby!!.

\section{Les aménagements périphériques}

Cette categorie regroupe des projets qui n'avaient pas comme préoccupation de départ les touristes, mais dont le succès a attiré ceux-ci. Ainsi donc, des équipements dont la vocation première était destinée à I'amélioration de la qualité de vie des rési. dents, deviennent parfois malgré eux des attractions touristiques fort populaires. C'est le cas, notamment, de plusieurs importants projets de rénovation urbaine. Le réseau souterrain de Montréal a, quant à lui, acquis une renommée internationale, mais l'idée de départ était de procurer aux Montréalais un moven d'affronter les rigueurs du climat, ou plutôt de les contourner. Lors de leur planification, cependant, ces projets doivent toujours tenir compte d'une certaine vocation touristique. II est bien évident que les projets de réaménagement du Vieux Port de Montréal ou de celui de Québec doivent faire une place importante aux touristes. De mëme, l'implantation de musées, de stades ou d'autres équipements socio-culturels ou sportifs comportent toujours une double dimension locale et touristique.

\section{Les concessions aux touristes}

Contrairement à la précédente catégorie, les projets de ce type niont absolument rien à voir avec le tourisme, ni de près ni de loin. Toutefois, en raison de leur importance, pour soigner l'image publique de l'entreprise, ou pour toute autre raison, il arrive que des projets acquièrent une dimension touristique, meme planifiée. C'est le cas, notamment, des barrages d'Hydro-Québec. II ne fait aucun doute que personne (qui saitl) ne serait assez farfelu pour faire édifier un barrage hydro-électrique à seule fin de montrer aux autres qu'on sait faire. Toutefois, lorsque l'oeuvre est parachevée, pourquoi la cacher, et surtout, pourquoi ne pas la mettre en vedette? Et c'est ainsi que le barrage Daniel Johnson est devenu une importante destination des clubs de l'Age d'or. 
Les aménagements "non-touristiques" A l'inverse des premiers types d'aménagements, ces derniers n'avaient au départ aucune vocation ni velléité touristique bien précise; et on n'a donc pas considéré cette dimension lors de leur conception et de leur planification. La tour de Pise, c'est bien connu, n'a pas été dessinée par un ingénieur en état d'ébriété. Serait-elle aussi populaire et aurait-elle surmonté les vicissitudes des ans dans une banale position verticale? D'autres accidents, même et surtout macabres, ont également une irrésistibilité qui comble d'aise certains touristes. En leur temps, le teu de Cabano ou les glissements de terrain de Nicolet ou de Saint-JeanVianney ont probablement attiré plus de touristes que tous les "prenez-le-tour-duQuébec" réunis. Dans ces derniers cas, on ne peut toutefois pas parler d'aménage: ments. Mais que penser, cependant, du pouvoir d'attraction que constituent les centres commerciaux comme les Galeries SaintBruno ou le Complexe Desjardins? Lorsque des autobus nolisés s'y rendent régulièrement, ne peut-on aे juste titre les classer compe attractions touristiques? et pourtant, il s'agit là d'une dimension qui avait sûrement échappé à leurs concepteurs.

Et, pour finir, bien sūr, la catégorie des "fours", ces aménagements conçus pour touristes exclusivement, mais qui n'ont pas su remplir leurs promesses. Que peut-on faire d'un éléphant rose? ? $^{[2]}$

\section{Les intervenants de l'aménagement}

Tous ces projets ou amenagements que nous avons passés en revue nécessitent un effort de planification. Nous savons que cette planification n'est pas toujours réalisée ou du moins pas toujours selon les meilleurs conditions. Parmi les types que nous venons d'énumérer, il s'en trouve mème dont la caractéristique est de ne pas avoir été spécifiquements planifiés pour les fins auxquelles ils servent en définitive. Au départ, deux ordres de problèmes confrontent donc ceux qui desirent apporter une approche rationnelle à la planification des aménagements touristiques. Le premier est d'ordre conjoncturel, et il est relié à une mauvaise interprétation ou encore aे une mauvaise prévision de la finalité du projet. Par ailleurs, lorsque la vocation touristique d'un projet se développe en cours de route. il est généralement assez facile de pallier au manque de prévision du départ. II ne s'agit donc pas à proprement parler d'un manque de planification. Quant au deuxième type de problème, il est relié a la nature mème des intervenants et résulte le plus souvent d'un manque de concertation au niveau des résultats.

Les intervenants responsables de la planification se retrouvent des deux cótés des pro-

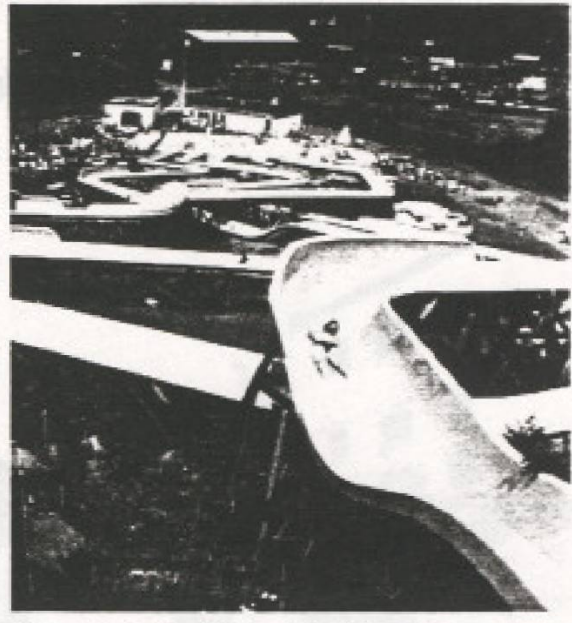

II s'agit là d'activités dites "foot loose" que I'on pourrait donc à la rigueur implanter $n$ 'im. porte oủ..

jets, soit à titre d'initiateurs, soit à titre d'agents de régulation ou de contrôle. Les premiers sont bien connus, et leur rôle de planification s'assimile à celui des promoteurs privés, qu'il s'agisse d'intervenants privés ou publics. II en va différemment, toutefois, du deuxième groupe. Dans le cadre du présent article, nous laisserons délibéré ment de cóté tous les autres intervenants pour nous en tenir aux agents de régulation et de contrôle.

Qui sont-ils, ces intervenants régulateurs? Au premier chef, mais loin des lieux du combat, se regroupent tous les organismes gouvernementaux qui pondent les règlements en vue de rendre opérationnelles les diverses lois reliées de près ou de loin à l'amé nagement. Plus près de la gestion quotidienne de l'espace, à l'autre extrémité, se retrouvent les municipalités et leur pouvoir de contröle sur l'émission, notamment, des permis de construction, assurant par là le respect des plans et règlements d'urba nisme. Les municipalités régionales de comté exercent également un contrỏle sur I'utilisation du sol. De plus, si l'on s'en tient aux propositions contenues dans le document de consultation sur le développement des régions,

"Bien que la MRC ne puisse constituer le seul interlocuteur en matiere de développement régional, le gouvernement du Québec reconnaît son importance. // entend donc appuyer par des contrats de développement le démarrage et la réalisation de projets intégrés de développement sur le territoire de la MRC. Ces projets pourraient s'inscrive dans le cadre du schéma d'amé. nagement de la MRC ou être proposés par des promoteurs ou des organismes locaux. "mal

Si on analyse les législations qui ont insufflé une nouvelle orientation aux préoccupations des planificateurs au Québec ces dernières années, il est deux lois qui apportent l'essentiel du cadre administratif et juridique dans lequel la planification des aménagements touristiques doit se concevoir. $\mathrm{Ce}$ sont la Loi 90 sur la protection du territoire agricole $^{(4)}$ et la Loi 125 sur l'aménagement et l'urbanisme ${ }^{|5\rangle}$. De plus, les décrets du ministère de l'Environnement, notamment sur la disposition des eaux usées, jouent egalement un rôle important de contrôle des activités de villégiature.

\section{La Loi sur la protection du territoire agricole}

Cette loi, en vigueur depuis décembre 1978 , a eu pour effet de répartir le territoire du Québec en zones blanches et en zones vertes. Les zones blanches sont celles où la loi ne prévoit aucune restriction tandis que les zones vertes sont assujetties à des normes de développement très strictes. De fait, dans les zones vertes, ou plus exactement dans les régions agricoles désignées, une personne ne peut, sans l'autorisation de la Commission de protection du territoire agricole, la CPTA, utiliser un lot à une fin autre que l'agriculture. Ainsi donc, à l'intérieur d'une zone agricole désignée, tout projet d'amé nagement touristique devra faire l'objet d'une autorisation de la part de la CPTA; et l'expérience a démontré que ces déroga tions sont excessivement rares. A titre d'exemple, il est bon de rappeler l'expé rience de la municipalité de Contrecoeur qui s'est rendue compte que l'on ne pouvait impunement contourner cette loi: la municipalité a dú procéder à la démolition d'une aréna qu'elle avait érigée à l'intérieur de sa zone agricole. La loi reconnait toutefois les droits acquis, et ceux-ci subsistent mème lors de l'interruption ou l'abandon de l'utilisation, sauf si le lot a été laissé sous couverture végétale pendant plus d'un an. De mème, la superficie du lot peut ètre portée à un hectare lorsqu'il s'agissait d'utilisations à des fins commerciales, industrielles ou institutionnelles.

Comme les zones blanches ne recouvrent que les territoires dejà urbanisés auxquels sont consenties des zones limitées d'expan sion ainsi que les zones vraiment impropres à l'agriculture, cette loi a des conséquences très contraignantes en ce qui a trait à la loca lisation d'activités touristiques. Ainsi, les terrains de camping du Domaine Rouville ou de Sainte-Madeleine, par exemple, n'auraient jamais vu le jour si la "loi 90" avait été en vigueur à l'époque. Par contre, aucun problème pour les projets d'expansion de la Station du Mont-Tremblant puisqu'elle n'est pas située en zone agricole.

Sur le plan de l'aménagement, les principaux effets de cette loi seront donc d'em. pècher l'expansion en zone agricole d'activités existantes (sauf jusqu'à concurrence d'un hectarel ou la venue d'activités para sitaires, de même que l'amenagement de projets nouveaux. Sur le plan de l'hébergement ou de la restauration, notamment, finis les relais de campagne ou les gites ruraux 
en zone verte. Finis également les lotissements de villégiature, les parcs à roulottes ou les campings dans ces mèmes zones. dans les zones blanches, soit à l'intérieur des périmètres urbains, soit en montagne ou encore dans certaines régions épargnées par la marée verte.

\section{La Loi sur l'aménagement}

\section{et l'urbanisme}

La principale caracteristique de cette loi sanctionnée en décembre 1979, fut la créa tion des municipalités régionales de comté. Les MRC se sont vues confier la responsabilité de l'élaboration des schémas d'aménagement, et partant, du contrôle de l'amé nagement à l'échelle régionale. Parmi ces fonctions d'aménagement, les propositions visant la redefinition des zones agricoles d'intéresser ceux qui sont aux prises avec ces zones. En effet, les MRC sont l'orga. nisme tout désigné pour infléchir les mem. bres de la CPTA, dans la mesure où il pourra être établi que les activités préconisees à l'encontre de l'agriculture s'inscrivent à l'intérieur d'un plan global d'aménagement du territoire à l'échelle d'une région donnée.

Les MRC étant responsables obligatoirement de la désignation des grandes utilisa tions du territoire et des infrastructures à caractère intermunicipal, tout projet d'envergure en matière d'amenagement touristique devra être conforme aux orientations du schema. A cet effet, des consultations devront être tenues avec tous les intervenants afin de procéder à une planification concertee. II importe ici de souligner que la loi prévoit également que les ministères sont jets d'amenagement.

Deux autres éléments de la loi méritent d'être soulignés: ce sont la réglementation de contrôle intérimaire et les plans d'urbanisme locaux. La réglementation de contröle intérimaire s'applique durant toute la phase d'élaboration du schéma d'aménagement; elle s'appliquera également à l'échelle locale Toutes ces activites devront se cantonner sont certes parmi celles qui risquent le plus tenus de faire part à la MRC de leurs pro-

durant la période d'élaboration des plans d'urbanisme. Elle a pour effet d'interdire toute nouvelle utilisation du sol ou toute nouvelle construction excepté notamment pour des fins agricoles, sauf dans les zones exclues du contrôle. A toutes fins utiles, durant la période d'élaboration des schémas et des plans, plusieurs zones seront astreintes à des controles ayant pour effet de geler le développement à l'intérieur de ces zones. II faut toutefois souligner que le règlement de contrôle interimaire est un outil evolutif et qu'il peut être modifie en tout temps. Les plans d'urbanisme existaient avant la venue de la Loi sur l'aménagement et l'urbanisme. Cette loi vient renforcer leur portée en les sanctionnant par un cadre législatif et administratif, sans toutefois les rendre obligatoires. Il est à prevoir que les municipalites, et même les plus petites, voudront à la suite de l'adoption du schéma d'aménagement de leur MRC d'appartenance adopter un plan et une réglementation d'urbanisme sur leur territoire, de façon à préciser et à contrôler leur cadre d'aménagement.

\section{Les règlements du ministère \\ de I'Environnement}

En plus des deux précedentes lois, les règle ments du ministère de l"Environnement du Québec viennent encore ajouter aux contrôles exercés sur le développement. Ainsi, des normes très strictes auxquelles doivent adherer tous les règlements régissent maintenant la taille minimale des lots à batti et ont été incorporées aux exigences du MAM pour ne pas désavouer les règlements de contrôle intermédiaire ${ }^{16 i}$ : dans le cas des lots non ou partiellement desservis, la superficie minimale est de 3000 mètres carres et mème de 4000 mètres carrés le long d'un cours d'eau ou en bordure d'un lac. La profondeur minimale d'un lot en bordure d'un plan d'eau est d'ailleurs fixee à 45 mètres dans le cas des lots desservis et elle est portée à 75 mètres pour les lots non ou partiellement desservis.

Ces normes visent évidemment la protection de l'environnement, mais elles auront des conséquences importantes sur le nou-

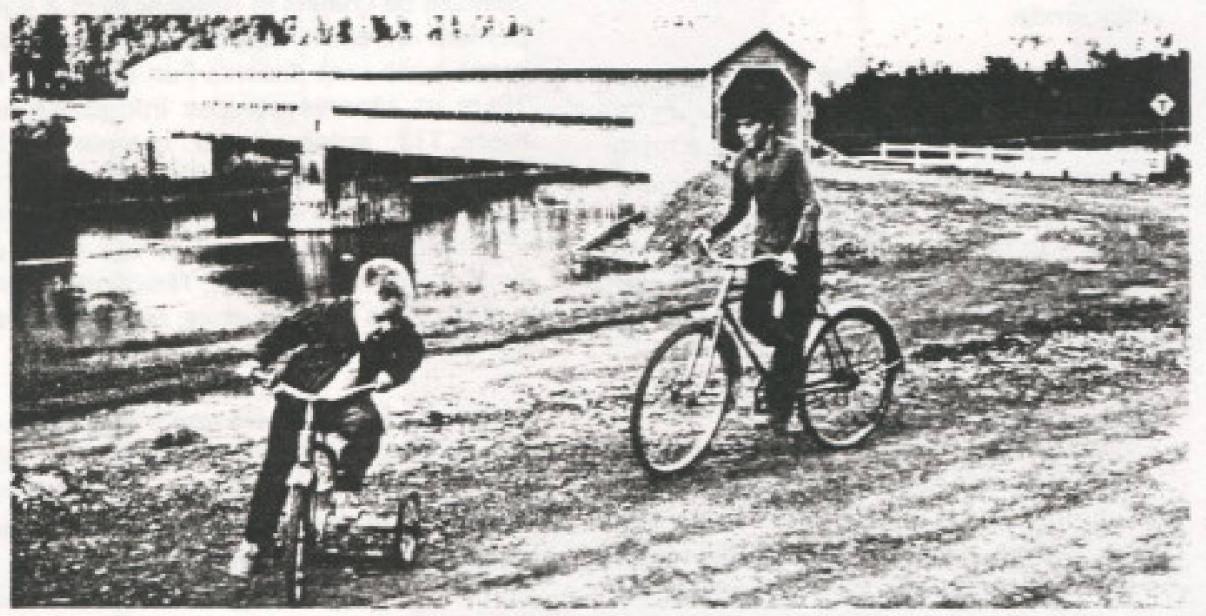

Ce pont n'a pas deté construit pour les touristes... et pourtant! veau visage des zones de villegiature. En raison de ces contraintes on est en droit de s'attendre à ce que les projets d'amenagement seront dorénavant dotés de services d'egoût et d'aqueduc, ce qui en fera haus. ser les coüts. De nouvelles formes d'ame nagements axées sur une plus grande densification en découleront fort probablement.

D'autres réglements ou interventions minis térielles font également en sorte que le developpement de la villegiature sera de plus en plus contrỏlé ou encadré. Ainsi, les règles d'occupation des terres de la Couronne sont maintenant beaucoup plus strictes.

Prudence et concertation, telles sont les deux vertus de l'aménagement touristique dans le contexte québécois depuis les changements que l'on vient de décrire. Prudence, certes, puisque les réglementations diverses qui se font de plus en plus sentir dans le milieu viennent, parfois de façon abrupte, mettre fin au laisser aller et meme au laxisme qui avaient cours en matière d'aménagement et ce, principalement en milieu rural ou périphérique. Concertation, aussi et sinon davantage, puisque les nouvelles règles du jeu placent l'arène directement sur le terrain, à l'échelle de la région, donc hors des officines centrales. Ainsi, les differrents acteurs se rencontreront sur place et auront l'occa sion de composer les uns avec les autres, alimentant ainsi la phase d'elaboration des schémas d'aménagement. C'est là qu'en principe seront effectues tous les arbitrages touchant les conflits d'usages ainsi que les prises de décision relatives aux grands travaux nécessaires au succès des dits projets d'aménagement.

La recente consultation du ministre délégué à l'Aménagement avec son document intitulé Le choix des régions, et les remous que sa publication a suscites, soulevent toutefois des interrogations sur les résultats tangibles de cette concertation au niveau des régions. La concertation étant un outil entre les mains de pouvoirs politiques, il parait en effet inevitable que les régions deviendront tout autant la scène de dissentions que d'acconds harmonieux. Esperrons que ces derniers l'emporteront! $f$

\section{Références}

1. DEMERS Jacques, Le tourisme en péril, editions Nowvelle optique, Montréal, 1983.

2. C'est ainsi, rappelons-le, que les publicistes de l'aeroport de Mirabel designent ce dernier

3. Gouvernement du Québec lle ministre Francois Gendranl Le choix des régions. Document de consultation sur le developpement des repions. 1983

4. Loi sur la protection du territaire egricole. LR.O. C. P.41.1

5. Loi sur l'aménagement et Vurbanisme, L.R.O. c. A. 19.1

6. Gouwernement du Ouebec, Miristere des Aftaires municipales, Direction génerale de rurbanisme - Le point sur toute la question du réglement de con trale intêrimaire de la municipalité regionale de Comte - actobre 1982 